University of Nebraska - Lincoln

DigitalCommons@University of Nebraska - Lincoln

The Optimal Bulk Wind Differential Depth and the Utility of the Upper-Tropospheric Storm-Relative Flow for Forecasting Supercells

\author{
Adam L. Houston \\ Richard L. Thompson \\ Roger Edwards
}

Follow this and additional works at: https://digitalcommons.unl.edu/geosciencefacpub

Part of the Earth Sciences Commons

This Article is brought to you for free and open access by the Earth and Atmospheric Sciences, Department of at DigitalCommons@University of Nebraska - Lincoln. It has been accepted for inclusion in Papers in the Earth and Atmospheric Sciences by an authorized administrator of DigitalCommons@University of Nebraska - Lincoln. 


\title{
The Optimal Bulk Wind Differential Depth and the Utility of the Upper-Tropospheric Storm-Relative Flow for Forecasting Supercells
}

\author{
AdAM L. Houston \\ Department of Geosciences, University of Nebraska_Lincoln, Lincoln, Nebraska \\ RichARD L. THOMPSON AND ROGER EDWARDS \\ Storm Prediction Center, Norman, Oklahoma
}

(Manuscript received 23 January 2007, in final form 13 February 2008)

\begin{abstract}
An analysis of $4 \mathrm{yr}$ of Rapid Update Cycle-2 (RUC-2) derived soundings in proximity to radar-observed supercells and nonsupercells is conducted in an effort to answer two questions: 1) over what depth is the fixed-layer bulk wind differential (BWD; the vector difference between the wind velocity at a given level and the wind velocity at the surface) the best discriminator between supercell and nonsupercell environments and 2) does the upper-tropospheric storm-relative flow (UTSRF) discriminate between the environments of supercells and nonsupercells? Previous climatologies of sounding-based supercell forecast parameters have documented the ability of the 0-6-km BWD in delineating supercell from nonsupercell environments. However, a systematic examination of a wide range of layers has never been documented. The UTSRF has previously been tested as a parameter for discriminating between supercell and nonsupercell environments and there is some evidence that supercells may be sensitive to the UTSRF. However, this sensitivity may be a consequence of the correlation between UTSRF and the surface to midtropospheric BWD. Accurately assessing the ability of the UTSRF to distinguish between supercell and nonsupercell environments requires controlling for the surface to midtropospheric BWD.

It is shown that the bulk wind differential within the $0-5-\mathrm{km}$ layer delineates best between supercell and nonsupercell environments. Analysis of the UTSRF demonstrates that even when not controlling for the BWD, the UTSRF has limited reliability in forecasting supercells. The lack of merit in using the UTSRF to forecast supercells is particularly evident when it is isolated from the BWD. Because the UTSRF and BWD are not independent, controlling for the BWD when examining the UTSRF reveals that the UTSRF is not a fundamental parameter that can be used to distinguish supercell from nonsupercell environments. Therefore, this work demonstrates that the UTSRF is an unreliable metric for forecasting supercell events.
\end{abstract}

\section{Introduction}

The work of Thompson et al. (2003, hereafter T03) represents perhaps the most ambitious attempt to date to develop a baseline of sounding-based forecast parameters for discriminating between supercells and nonsupercells. Any sounding-based climatology intended to identify parameters that correctly distinguish supercell from nonsupercell environments will be prone to errors resulting from the inability of soundings to accurately represent the storm-scale environment and

Corresponding author address: Dr. Adam L. Houston, Dept. of Geosciences, University of Nebraska-Lincoln, 214 Bessey Hall, Lincoln, NE 68588.

E-mail: ahouston@unl.edu from inaccuracies in methods used to determine if a storm is a supercell. Uncertainties in the degree to which soundings represent particular storms/events can only be mitigated, never removed, but the T03 climatology avoids the liberal proximity criteria implemented in previous climatologies (e.g., Rasmussen and Blanchard 1998, hereafter RB98; Craven et al. 2002) by using Rapid Update Cycle-2 (RUC-2; Benjamin et al. 2004) analysis soundings whose temporal and spatial coverage far exceed those of the rawinsonde observations used previously. The T03 climatology also uses radar observations instead of proxies to characterize storms as either supercells or nonsupercells (in contrast, RB98 used reports of hail diameters greater than or equal to $10 \mathrm{~cm}$ to identify supercells). Using these approaches, T03 were able to robustly test the ability of 
sounding-based parameters to discriminate between the environments of supercells and nonsupercells. Moreover, their work produced a soundings database that enables further interrogation of existing parameters and/or the development of new ones.

The T03 database along with supplemental soundings collected by Thompson et al. (2007; hereafter TME07) are used herein to address two outstanding questions pertaining to sounding-based forecast parameters used previously to discriminate between the environments of supercells and nonsupercells:

1) Over what depth is the vertical bulk wind differential (the vector difference between the wind velocity at a given altitude and the wind velocity at the surface) the best discriminator between supercell and nonsupercell environments?

2) Does the upper-tropospheric storm-relative flow discriminate between the environments of supercells and nonsupercells?

The question of optimal bulk wind differential (BWD; often simply referred to as vertical shear in the literature) depth may seem moot considering the documented ability of the $0-6-\mathrm{km}$ BWD in discriminating between supercells and nonsupercells (T03; RB98). However, an examination of the ideal depth for the BWD using a wide range of possible layers has never been documented; thus, it remains unclear if the 0-6$\mathrm{km}$ layer is indeed optimal. TME07 did examine the optimal depth of the effective BWD (EBWD) using a wide range of storm depth fractions. The EBWD is the vector wind difference computed over a percentage of the total storm depth defined as the layer between the effective inflow base and the most unstable parcel equilibrium level. The advantage of the EBWD over the BWD is that fixed layers may not be versatile enough to account for environments that support anomalously deep or anomalously shallow storms or that support elevated storms for which the surface wind is less relevant or irrelevant to the storm mode. The limitation of the EBWD is that it requires a complete vertical profile of thermodynamic data. Thus, when only kinematic data are available or when the vertical resolution of thermodynamic data is too coarse, the BWD is a better alternative but should be used cautiously when the environments should support anomalously deep, anomalously shallow, or elevated storms.

The sensitivity of supercells to upper-tropospheric storm-relative winds has been examined by RB98 but has yet to be tested using the superior T03 sounding database. Moreover, RB98 acknowledged that the sensitivity of supercells to the upper-tropospheric storm- relative flow may be a direct consequence of the correlation between this parameter and the surface to midtropospheric BWD, which distinguishes well between supercells and nonsupercells. Accurately assessing the ability of the upper-tropospheric storm-relative flow to distinguish between supercells and nonsupercells requires controlling for the surface to midtropospheric BWD.

This article proceeds in section 2 with an explanation of the data and techniques used for the analysis. Results from the examination of BWD depth and uppertropospheric storm-relative winds are reported in sections 3 and 4, respectively, followed by a summary of conclusions in section 5.

\section{Data and methodology}

The soundings of the T03 database have been constructed from the analysis data of the RUC- 2 model. Each sounding represents the inflow environment attributed to a specific storm that has been classified as either a supercell or nonsupercell based on Weather Surveillance Radar-1988 Doppler (WSR-88D) reflectivity and velocity data. T03, Markowski et al. (2003), and TME07 have determined that RUC-2 analysis soundings collected in this manner sufficiently represent the environments of the storms to which they are attributed. All data were collected during two periods: April 1999-June 2001 and March 2003-March 2005. Data collection techniques during these two periods were virtually identical to the methodology documented by T03, but subtle differences will be explained below. In total, 250 nonsupercell soundings and 829 supercell soundings were used for this analysis.

Synthesis of this dataset began with storm identification and classification. For a storm to be characterized a supercell it must satisfy all of the following criteria: 1) a hook echo and/or inflow notch must be present, 2) the peak cyclonic azimuthal shear must be $\geq 20 \mathrm{~m} \mathrm{~s}^{-1}$ across a distance of $10 \mathrm{~km}$ or less at elevation angles of $0.5^{\circ}$ or $1.5^{\circ}$, and 3 ) cyclonic shear must persist for at least $30 \mathrm{~min}$. A nonsupercell is identified when a discrete storm possesses a maximum composite reflectivity of $40 \mathrm{dBZ}$ for more than $30 \mathrm{~min}$ but failed to meet any of the supercell criteria listed above. ${ }^{1}$ Following storm identification the time and location at which each supercell was at its strongest was identified. Supercell in-

\footnotetext{
${ }^{1}$ The "marginal" category used by T03 is not included in this analysis.
} 


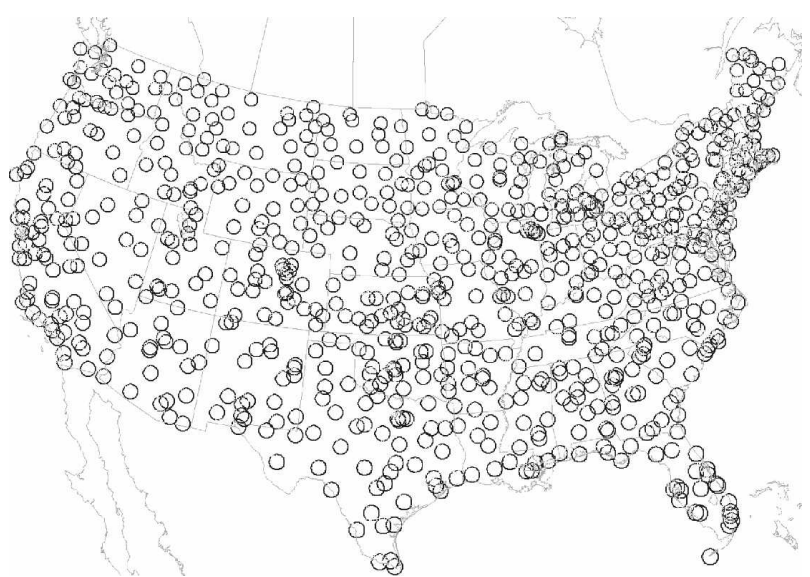

FIG. 1. Locations of all possible "point forecast soundings" used during the second data collection period.

tensity was gauged according to the highest F-scale rating assigned to tornadoes that it produced; or, in the absence of tornadoes, the largest hail diameter that the storm produced; or, in the absence of both hail and tornadoes, the magnitude of the cyclonic shear or the prominence of the reflectivity signatures. If multiple supercells existed within $3 \mathrm{~h}$ and/or $185 \mathrm{~km}$ of one another, then only the most intense supercell in the group was recorded. This was done to avoid biasing the dataset toward single events with large numbers of supercells. Because of this filtering, it is most accurate to interpret this dataset as a collection of supercell events and nonsupercell events.

The method used for attributing soundings to storms differed slightly between the two data collection periods. During the first collection period (April 1999-June 2001), RUC analysis data were bilinearly interpolated to the location of the nearest upwind surface weather observation station within $40 \mathrm{~km}$ and $30 \mathrm{~min}$ of the location and time of maximum storm intensity. In the second data collection period, only predetermined point forecast sounding locations were used (see Fig. 1 for an illustration of all possible locations). These locations were chosen to coincide with selected raob, wind profiler, and/or surface observation sites. A point forecast sounding at one of these locations was attributed to a particular storm if the storm passed within $40 \mathrm{~km}$ of a sounding site within $30 \mathrm{~min}$ of the analysis time. Because fewer potential sites were available during the second data collection period, some soundings were associated with storms that were not at peak intensity while within $40 \mathrm{~km}$ of the sounding site and $30 \mathrm{~min}$ of the analysis time. However, differences in representativeness between the two collection periods are likely small. In fact, results from T03 can largely be replicated using only the data from the second collection period.
For both data collection periods, soundings containing no surface-based CAPE were removed from the dataset as the associated storms were presumed to be "elevated" (sustained with air from above the boundary layer). It should also be reiterated that only cyclonic supercells were analyzed in T03 and for this study. Data for anticyclonic supercells continue to be collected and will be analyzed separately once their sample size becomes more robust.

\section{Bulk wind differential}

\section{a. Background}

While the climatologies of RB98 and T03 have documented the ability of the 0-6-km BWD to discriminate between supercellular and nonsupercellular environments, a systematic examination of a wide range of alternative layers has not been documented. Thus, it is uncertain if the 0-6-km layer is actually the best representation of the environments of the two storm types. Before proceeding to examine additional layers, it is worth probing the theoretical and historical merit in using the 0-6-km BWD/vertical shear.

Since the early observational studies of Fawbush and Miller (1952, 1954) and Fawbush et al. (1957), it has been clear that the likelihood of "significant" tornadoes is correlated with the magnitude of the vertical wind shear. In as far as significant tornadoes serve as proxies for supercells, their climatologies revealed that supercells tend to be favored in environments where the winds increase and veer through $500 \mathrm{mb}$. However, it is unclear from their work if the 500-mb level was chosen out of convenience or because it proved to be superior to other levels in discriminating between environments that are capable of supporting significant tornadoes and environments that are not. In fact, Marwitz (1972) later argued that it is the vertical shear through the entire cloud column that must be considered when discriminating between supercells and other storm types.

The use of vertical shear within the 0-6-km layer for forecasting supercells was first explored by Weisman and Klemp $(1982,1984)$. Largely based on the theoretical arguments for steady-state (supercellular) deep convection posited by Moncrieff and Green (1972), they asserted that one of the essential mechanisms controlling the distinction between supercells and nonsupercells was the balance between the inflow kinetic energy and the CAPE. Since the mean wind in the $0-6-\mathrm{km}$ layer often is considered a good proxy for storm motion, they proposed that the vector difference between the boundary layer and mean $0-6-\mathrm{km}$ winds could serve as a measure of the inflow kinetic energy. They also argued that because the difference between the mean 
0-6-km wind and low-level wind served as a measure of the wind shear, this parameter also captured the ability of the updraft to generate rotation; an essential distinction between supercells and nonsupercells. Weisman and Klemp $(1982,1984)$ incorporated this parameter into the denominator of the bulk Richardson number:

$$
\mathrm{BRi}=\frac{\mathrm{CAPE}}{\frac{1}{2}\left(\left|\overline{\mathbf{v}}_{6 \mathrm{~km}}-\overline{\mathbf{v}}_{\mathrm{BL}}\right|\right)^{2}},
$$

where $\overline{\mathbf{v}}_{6 \mathrm{~km}}$ is the density-weighted mean wind over the lowest $6 \mathrm{~km}$ and $\overline{\mathbf{v}}_{\mathrm{BL}}$ is the mean boundary layer wind (typically over the lowest $500 \mathrm{~m}$ ).

McCaul (1991) was the first to apply the kinematic portion of the BRi [the denominator of (1), referred to as the bulk Richardson number shear] to tornado environments. Shortly thereafter, Droegemeier et al. (1993) used the BRi shear in their analysis of numerically simulated deep convection showing that the parameter exhibited a high correlation with maximum vertical vorticity and recommending its use in predicting storm type.

The first documented application of the wind shear computed between the surface and $6 \mathrm{~km}$ (the bulk wind differential) without the layer averaging used in the bulk Richardson number shear can be attributed to RB98. RB98 concluded that the $0-6-\mathrm{km}$ BWD could be used in distinguishing between environments supportive of storms that produce significant (F2 or greater) tornadoes and/or hail 2 in. or larger in diameter (their "TOR" and "SUP" categories) and environments supportive of storms that produce lightning but no severe weather (their "ORD" category). T03 also applied the 0-6-km BWD to their samples of supercells and nonsupercells and concluded that "the $0-6 \mathrm{~km}$ [BWD] clearly discriminates between all supercells and nonsupercells, with no overlap in values between the 10th percentile for supercells ... and the 90th percentile for nonsupercells."

The 0-6-km BWD clearly differentiates between supercell and nonsupercell environments but some have suggested that vertical layers other than $0-6 \mathrm{~km}$ should be considered. As noted above, Marwitz (1972) posited that the vertical shear over the entire cloud column should be used. Rasmussen and Wilhelmson (1983) considered the mean shear (alternatively referred to as the hodograph length) in the 0-4-km layer to compare the environments of tornadic supercells, nontornadic supercells, and nonsupercells. Although their sample was rather small, they were able to demonstrate that the $0-4-\mathrm{km}$ mean shear might be capable of distinguishing between the different environments. Weisman and
Klemp (1986) conducted a set of numerical experiments in which the depth of the vertical shear layer was varied. Their numerical experiments revealed that shear across layers between $0-5$ and $0-7.5 \mathrm{~km}$ was more important for delineating supercell from nonsupercell environments than the vertical shear over a shallow 0-2.5-km layer alone.

Ultimately, the documented ability of the BWD parameter in delineating supercell from nonsupercell environments along with indications from both observational and numerical studies that layers other the 0-6$\mathrm{km}$ layer might accentuate this delineation indicate that a comparison of the separations between supercell and nonsupercell samples for a wide range of (fixed layer) BWD depths is necessary. Such a comparison has not been documented.

\section{b. Results}

Twelve layers were considered in this analysis. A summary of the results is illustrated in Fig. 2 as boxand-whiskers plots for the supercell and nonsupercell samples. Through visual inspection it is clear that the largest separation between distributions exists for layers whose upper bound resides in the midtroposphere (0-4 to $0-7 \mathrm{~km})$. To quantify the separation, the difference between the 10th percentile value of the supercell distribution and the 90th percentile value of the nonsupercell distribution $\left(S_{10}-N_{90}\right)$ along with the difference between the 5 th percentile value of the supercell distribution and 95th percentile value of the nonsupercell distribution $\left(S_{5}-N_{95}\right.$; not illustrated in Fig. 2) were computed for each layer. Results are plotted in Fig. 3. The differences between the sample separations for the 0-4- through 0-6-km layers are rather small and indicate that the BWD computed within any of these layers discriminates well between supercell and nonsupercell environments. Nevertheless, the largest $S_{10}-N_{90}$ separation exists for the $0-5-\mathrm{km}$ layer while the largest $S_{5}-$ $N_{95}$ separation occurs in the $0-5.5-\mathrm{km}$ layer. A nearly identical value of $S_{5}-N_{95}$ separation is evident for the 0-4.5-, 0-5-, and 0-5.5-km layers. Because of the large sample sizes used for this work, a $t$ test applied to each of the shear layers illustrates that differences between the supercell and nonsupercell samples are statistically significant at confidence levels near $100 \%$ for all shear layers. Nevertheless, the $t$ statistic, the number of standard deviations (from the mean) above/below which the means of the two samples would be equal (the null hypothesis), does provide further evidence that the best delineation between supercell and nonsupercell environments exists for the 0-4- to 0-5-km layers (Fig. 4). Further analysis of the $0-5-\mathrm{km}$ layer reveals that, 93\% of all supercells in the dataset occur in environments 

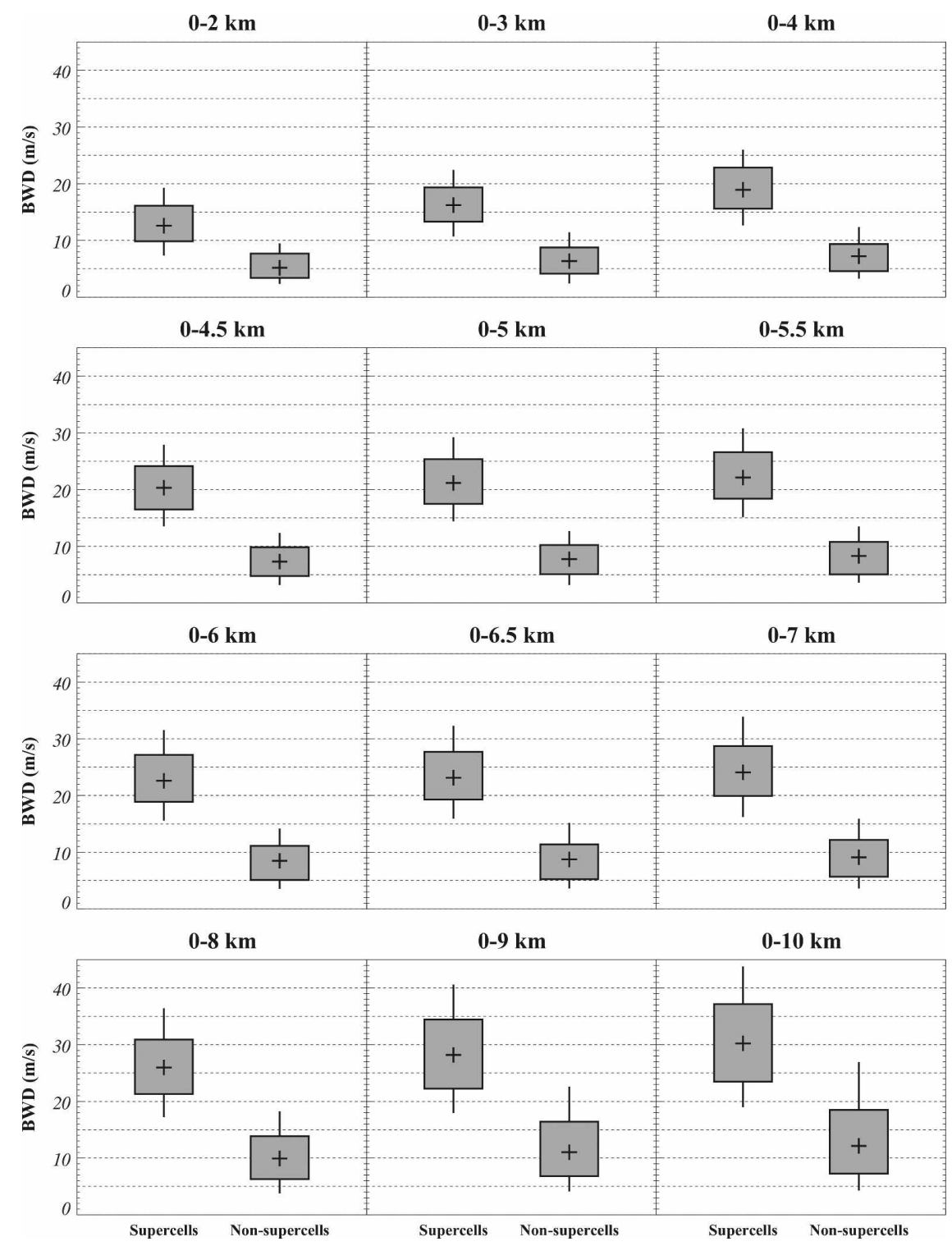

FIG. 2. Box-and-whisker plots for each of the 12 BWD layers analyzed. The shaded boxes indicate the bounds on the 25th-75th percentiles, the whiskers extend to the 10th and 90th percentiles, and the plus signs indicate the median values.

with BWDs greater than $13.5 \mathrm{~m} \mathrm{~s}^{-1}$ while $93 \%$ of all nonsupercells occur in environments with $0-5-\mathrm{km}$ BWDs of less than $13.5 \mathrm{~m} \mathrm{~s}^{-1}$. Ultimately this analysis demonstrates that, even though the $0-6$-km layer exhibits the large distribution separation (particularly relative to deeper layers) noted by T03 and RB98, it is the BWD within the $0-5-\mathrm{km}$ layer that delineates best between supercell and nonsupercell environments.

In their analysis of the EBWD, TME07 found that the best discrimination between supercells and nonsupercells existed for EBWD values computed over $40 \%$ of the storm depth. At these depths, the value of $S_{10}-N_{90}$ for EBWD was $+1.3 \mathrm{~m} \mathrm{~s}^{-1}$ (see their Fig. 10). The $S_{10}-N_{90}$ difference for the 0-5-km BWD presented here is $+1.7 \mathrm{~m} \mathrm{~s}^{-1}$. Thus, the computationally simpler BWD discriminates between supercell and nonsupercell environments as well as if not better than the EBWD. However, as stated in the introduction, in environments supportive of anomalously tall, anomalously short, or elevated storms, the EBWD should be used, if possible, in lieu of the BWD.

The most accurate assessment of the ability of a particular parameter to delineate between supercell and nonsupercell environments should be based on deter- 


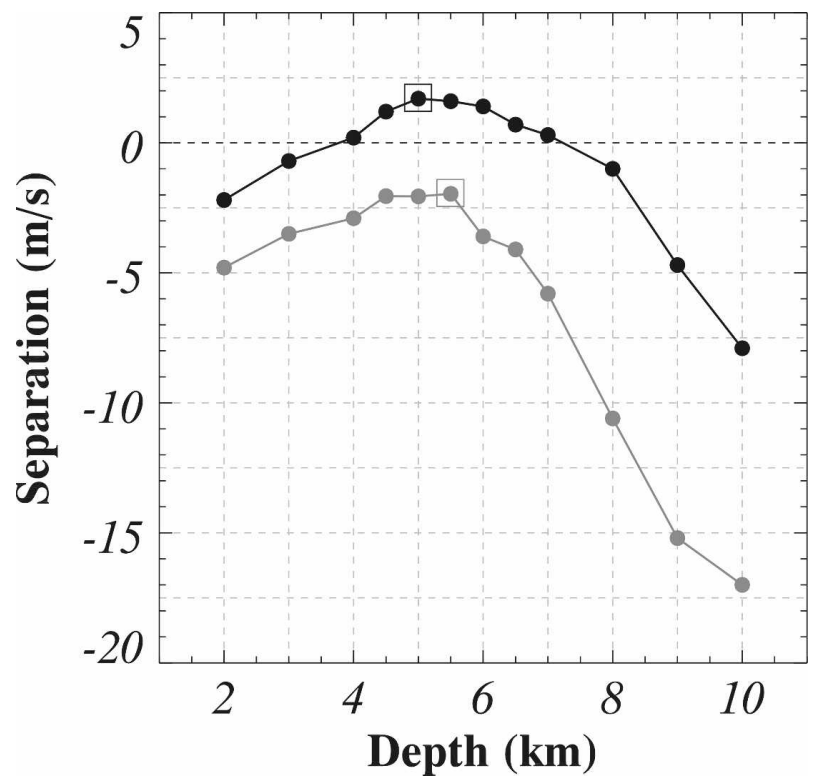

FIG. 3. Separations between the 10th percentile of the supercell sample and the 90th percentile of the nonsupercell sample $\left(S_{10}-\right.$ $N_{90}$; black curve) and between the 5 th percentile of the supercell sample and the 95th percentile of the nonsupercell sample $\left(S_{5}-\right.$ $N_{95}$; gray curve) for each BWD depth. Small squares indicate the BWD depth with the largest separation.

mining the likelihood of finding a supercell event among the entire population of supercells and nonsupercells compared to the likelihood of finding a nonsupercell event. While the samples of supercells and nonsupercells in this dataset are likely to be accurate representations of the complete populations of supercell events and nonsupercell events during the time period, it is unlikely that the ratio of the number of supercell events to the number of nonsupercell events $(829 / 250 \approx$ $3 / 1$ ) is representative of the actual ratio. Therefore, this type of analysis is not possible using this dataset unless it is modified to account for the actual ratio of supercell to nonsupercell events. Unfortunately, this ratio is unknown.

This limitation of the dataset does not undermine the analysis conducted here because the ability of a given parameter to differentiate between supercell and nonsupercell environments relative to another parameter is independent of the actual ratio of supercell to nonsupercell events. However, threshold values separating the two distributions will change. Consider for example the histograms illustrated in Fig. 5. At 0-5-km BWD values greater than a threshold of $\sim 12 \mathrm{~m} \mathrm{~s}^{-1}$, the likelihood of finding a supercell in the full dataset is higher than the likelihood of finding a nonsupercell in the full dataset. However, if the actual ratio of supercell to nonsupercell events is smaller than the 3-1 ratio reflected in this dataset, then, as long as the overall shapes of the

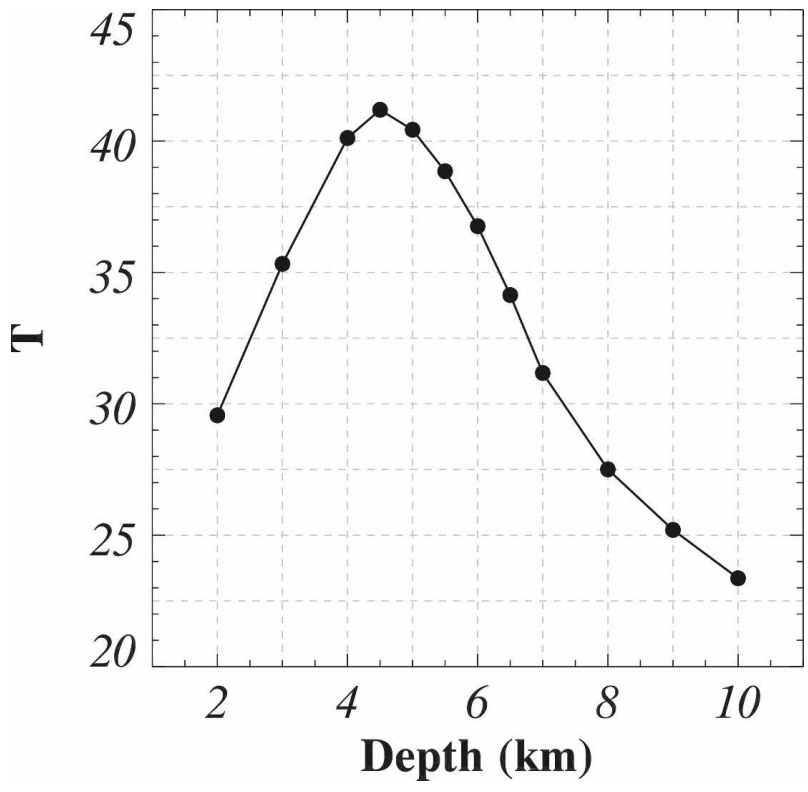

FIG. 4. The $T$-statistic values calculated for each BWD depth.

distributions remain the same, the threshold value would be larger than $12 \mathrm{~m} \mathrm{~s}^{-1}$.

To approximate the threshold value separating environments that are more likely to support supercell events from those that are more likely to support nonsupercell events, the $0-5-\mathrm{km}$ BWD distribution is reevaluated using supercell to nonsupercell ratios ranging from 3-1 (as represented in this dataset) to 1-20. The ratios are implemented by applying a constant scaling to the nonsupercell distribution of this dataset while retaining the median and standard deviation. (Thus for a 1-20 ratio, the number of nonsupercell environments in the dataset is multiplied by a scaling factor of $\sim 60$.) The threshold will be quantified by evaluating the probability of a supercell event within a given range (bin) of BWD values. This quantity is expressed as follows:

$$
100 \cdot \frac{\mathrm{ns}\left(m_{B}, R_{B}\right)}{\mathrm{ns}\left(m_{B}, R_{B}\right)+\operatorname{nns}\left(m_{B}, R_{B}, A\right)},
$$

where $\mathrm{ns}\left(m_{B}, R_{B}\right)$ is the number of supercell environments for a BWD midpoint value and range of $m_{b}$ and $\pm R_{B}$, respectively, and nns is the number of nonsupercell environments scaled by a factor $A$ (only the number of nonsupercells is adjusted to account for the supercell to nonsupercell ratio so the variable $A$ does not appear as a variable for ns). For values of (2) equal to (greater than) $50 \%$, the likelihood of finding a supercell event is equivalent to (greater than) the likelihood of finding a nonsupercell event.

Values of (2) are plotted as a function of $m_{B}$ in Fig. 6 for 0-5-km BWD at selected supercell to nonsupercell 


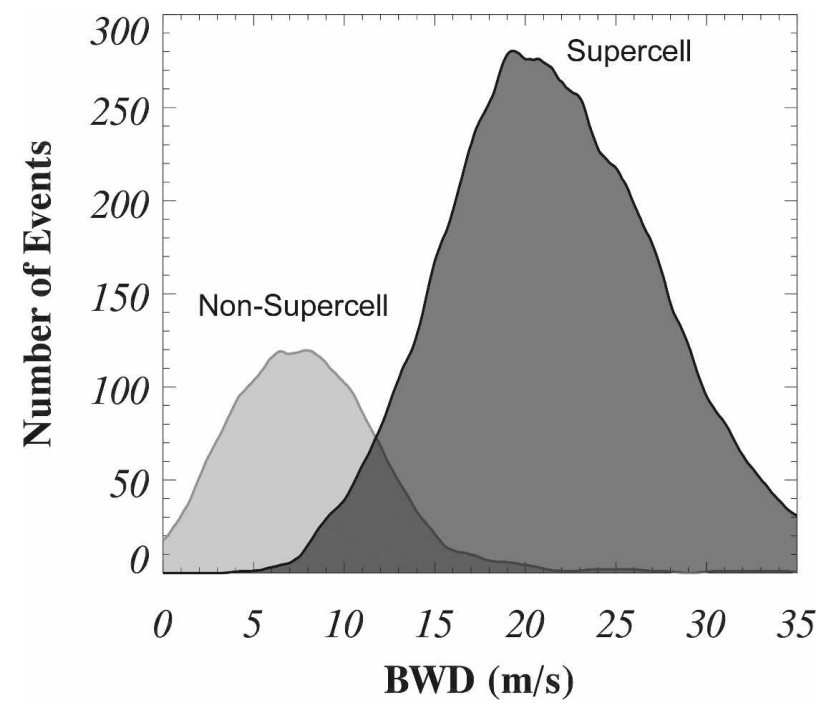

FIG. 5. Histograms of supercells (black curve and dark gray shading) and nonsupercells (dark gray curve and light gray shading) for the 0-5-km BWD. Histograms are developed using a bin size of $5 \mathrm{~m} \mathrm{~s}^{-1}$ (range of $\pm 2.5 \mathrm{~m} \mathrm{~s}^{-1}$ ), a bin midpoint spacing of $0.01 \mathrm{~m} \mathrm{~s}^{-1}$, and a boxcar smoother to remove stair-stepping and high-frequency variability.

ratios. For this analysis, $R_{B}$ is set to $\pm 2.5 \mathrm{~m} \mathrm{~s}^{-1}$, midpoint increments are $0.01 \mathrm{~m} \mathrm{~s}^{-1}$, and a boxcar smoother is applied to remove stair-stepping and high-frequency variability that is insignificant. The threshold value separating environments that are more likely to support supercells from those that are more likely to support nonsupercells is clearly dependent on the ratio of supercell to nonsupercell events. If the actual ratio is $1-1$, then the threshold value of $0-5-\mathrm{km}$ BWD would be $\sim 14$ $\mathrm{m} \mathrm{s}^{-1}$. However, if the actual ratio is closer to $1-20$, then the threshold would be $\sim 20 \mathrm{~m} \mathrm{~s}^{-1}$. There is informal evidence to suggest that the actual ratio may be between 1-10 and 1-20. Forecasters at the National Oceanic and Atmospheric Administration (NOAA)/ Storm Prediction Center have found success forecasting supercells when using a threshold of $\sim 18 \mathrm{~m} \mathrm{~s}^{-1}$ for the 0-6-km BWD.

Despite the variability in thresholds illustrated in Fig. 6 , for each ratio, supercells are more likely than nonsupercells for "large" BWD values (i.e., BWD values exceeding the particular threshold). As will be shown in the next section, the same conclusion cannot be made for the upper-tropospheric storm-relative winds.

\section{Upper-tropospheric storm-relative winds}

\section{a. Background}

RB98 have suggested that supercells may be favored over nonsupercells in environments with "strong"

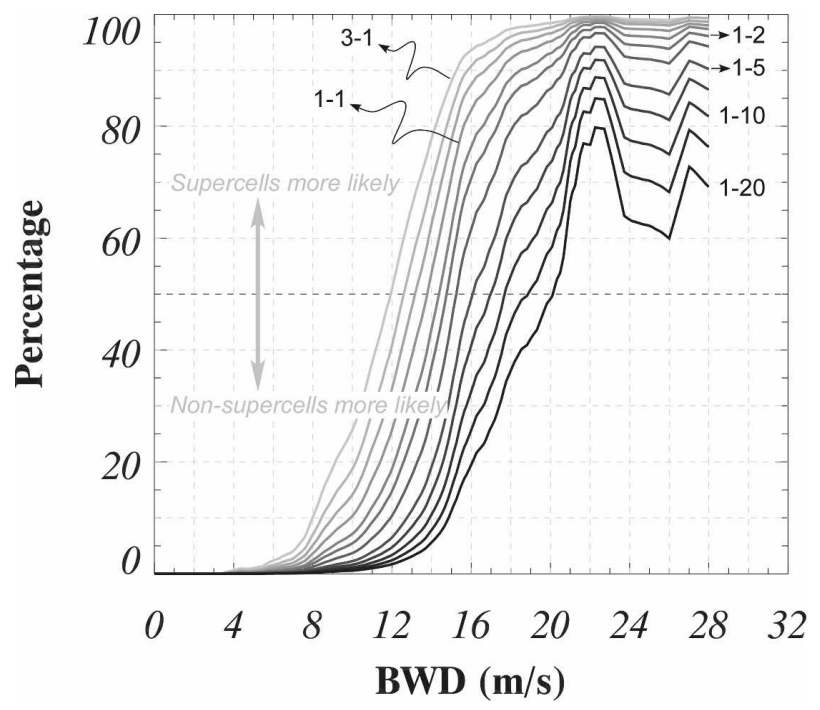

FIG. 6. Probability of a supercell for the $0-5-\mathrm{km}$ BWD. The abscissa represents the midpoint values of BWD and the ordinate represents the likelihood of finding a supercell in a given BWD. Shading of curves (from light gray to black) increases with increasing supercell to nonsupercell ratio. Ratios from 3-1 to 1-20 are illustrated.

storm-relative upper-tropospheric winds. They argued that stronger anvil-level flow promotes the evacuation of water mass accumulating near the storm summit and therefore would tend to support the updraft/mesocyclone longevity often considered a defining characteristic of supercells. This conjecture has some support in their climatology of "supercell" and "ordinary" storms. But since the bulk wind differential and uppertropospheric storm-relative flow (UTSRF) are not independent parameters (both are completely determined by the wind field), they caution that the separation of the supercell and ordinary categories using the UTSRF may be an ancillary consequence of the strong discriminatory ability of the surface to midtropospheric BWD. The purpose of this analysis is to evaluate the discriminating ability of the UTSRF while isolating it from the surface to midtropospheric BWD.

\section{b. Methodology}

The equilibrium level (EL) computed using the most unstable CAPE was chosen to represent the upper troposphere. Wind data were linearly interpolated to the EL when necessary. Because observed storm motion is not available before an event, forecasted storm motion must be used operationally and is thus incorporated herein to compute the storm-relative flow. Moreover, because storm mode is unknown a priori, supercell motion is assumed for all storms. Predictions of storm mo- 


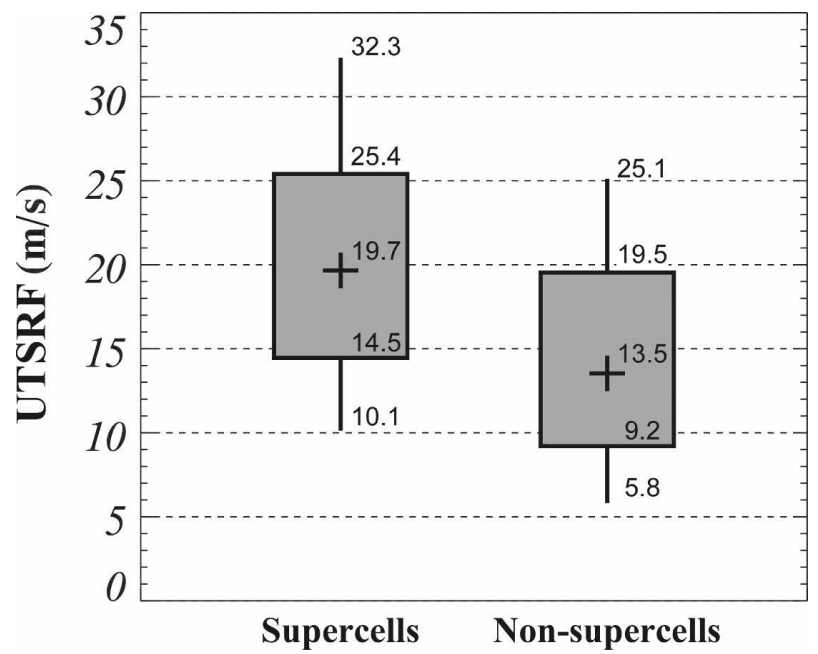

FIG. 7. As in Fig. 2 but for the upper-tropospheric stormrelative flow and with the annotation of values at the 10th, 25th, 50th, 75th, and 90th percentiles.

tion are prone to large errors (Ramsay and Doswell 2005), but the internal dynamics (ID) method (Bunkers et al. 2000) has been shown to perform well under most situations (TME07) and will be used here. (A portion of the analysis presented in the following subsection has been repeated using observed storm motions and appears in the appendix. The conclusions reached using observed storm motion are no different than the conclusions reached using predicted storm motion.)

\section{c. Results}

Consistent with the results of RB98, the supercells of this dataset tend to form in environments with UTSRF that is stronger than that of nonsupercellular environments (Fig. 7). ${ }^{2}$ However, the value in using this metric to forecast storm type is questionable considering the significant overlap in the distributions. Moreover, as stated above, the separation between the distributions may be a consequence of the sensitivity of the distributions to the surface to midtropospheric BWD and not because the UTSRF is an essential distinguishing metric.

One method for quantifying the relationship between BWD and storm-relative flow is with the linear correlation coefficient $(r)$. The values of $r$ for the UTSRF and several BWD depths, computed using all storms in the dataset, are listed in Table 1. It is clear that the UTSRF and BWD are positively correlated through the

\footnotetext{
${ }^{2}$ These differences are statistically significant above the $99 \%$ confidence level.
}

TABLE 1. Linear correlation coefficients $(r)$ between the BWD and the UTSRF.

\begin{tabular}{cc}
\hline \hline BWD depth $(\mathrm{km})$ & $r$ \\
\hline 4.0 & 0.25 \\
5.0 & 0.34 \\
6.0 & 0.40 \\
7.0 & 0.45 \\
8.0 & 0.53 \\
9.0 & 0.60 \\
10.0 & 0.66 \\
\hline
\end{tabular}

depths considered and that the correlation increases with increasing depth. This latter behavior is to be expected considering that BWD-layer depths approaching $10 \mathrm{~km}$ should become progressively less independent from the UTSRF. This finding provides some support for the assertion that the correlation between supercell probability and UTSRF is largely a consequence of the dependence of supercells on surface to midtropospheric BWD.

Unfortunately, the linear correlation coefficient fails to illuminate the sensitivity of supercells to the UTSRF with the sensitivity to BWD removed. To do this, the BWD must be controlled for while examining the likelihood of supercells as a function of the storm-relative flow. For an infinitely large dataset, this analysis would proceed by collecting only those soundings within the supercell and nonsupercell distributions that have a specific value of BWD. However, few if any actual environments in the dataset will have a BWD that is identical to the chosen value; so a finite range of BWD values must be used. Because of the sharp separation between the BWD associated with the supercell and nonsupercell distributions, this range of BWD values needs to be large enough to capture enough samples from both distributions to make the statistics meaningful. For this analysis a range of $\pm 2.5 \mathrm{~m} \mathrm{~s}^{-1}$ was chosen.

Figure 8 illustrates four subsamples from the supercell and nonsupercell datasets for which BWD values are within the narrow range of values specified above. This enables analysis of the supercell and nonsupercell distributions as a function of UTSRF while controlling for the BWD. It is clear from this figure that, compared to the distributions when not controlling for the BWD (light gray box-and-whisker plots duplicated from Fig. 7), the discriminating ability of the UTSRF is greatly diminished when controlling for BWD computed over depths greater than $4 \mathrm{~km}$ (note the increased overlap in the distributions for the 0-5-, 0-6-, and 0-7-km layers). As observed above, this degradation in the discriminating ability of UTSRF as the BWD-layer depth increases from 4 to $7 \mathrm{~km}$ is likely a consequence of the decreased 


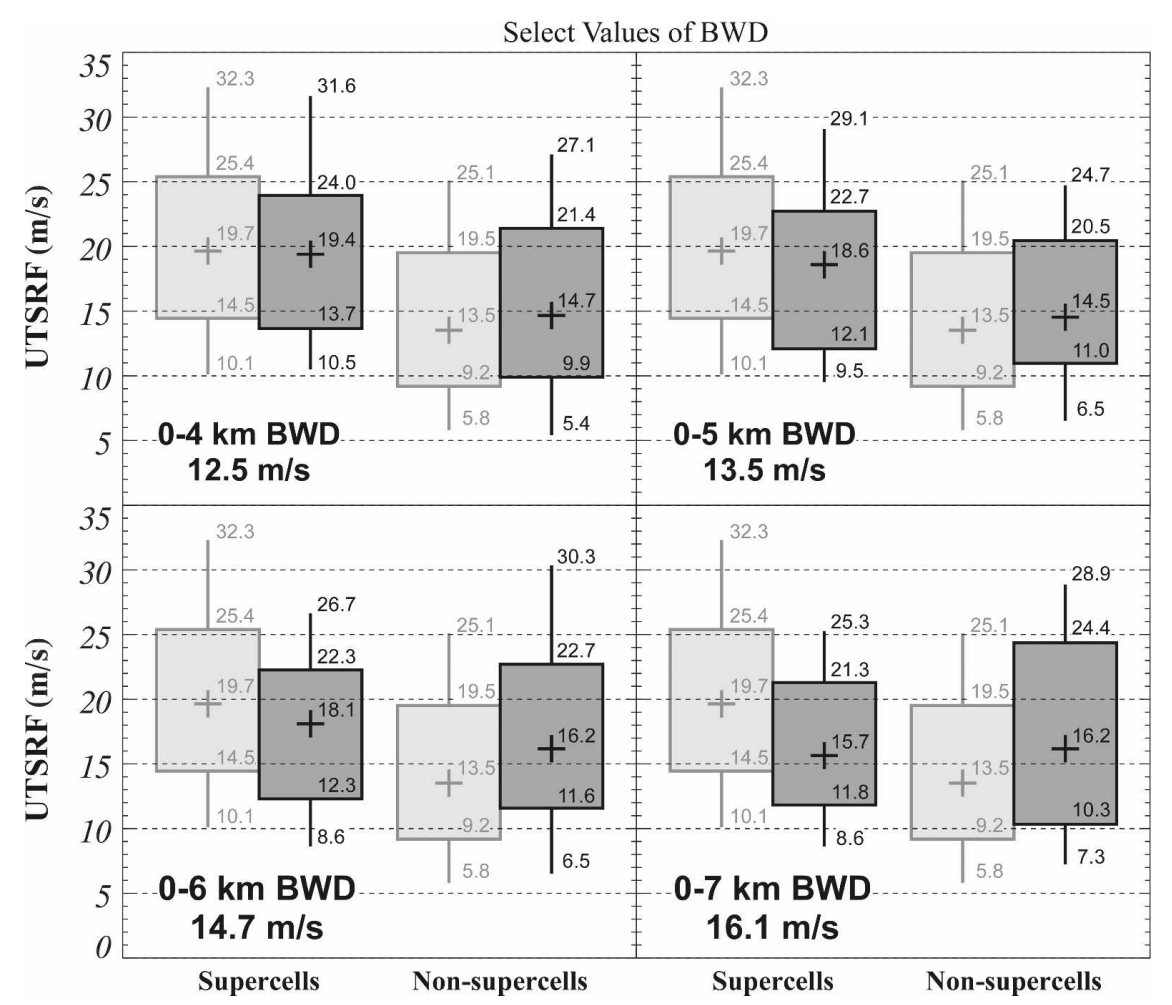

FIG. 8. As in Fig. 7 except that only specific ranges of BWD are considered (the BWD layer and midpoint value of the BWD range are listed at the bottom left of each panel). Light gray boxes are the distributions for all BWD values as illustrated in Fig. 7.

independence of the UTSRF and BWD parameters. Thus, this analysis provides a more compelling argument that the discriminating ability of UTSRF is largely tied to the discriminating ability of the BWD.

The large overlap in supercell and nonsupercell distributions for UTSRF, particularly when controlling for BWDs over layers of 5,6, and $7 \mathrm{~km}$, suggests that when isolated from the BWD, the UTSRF may not be a reliable metric for discriminating between environments that support supercells and environments that support nonsupercells. Unfortunately, with the exception of obvious distribution separations for parameters like BWD (cf. Fig. 2), the "largeness" or "smallness" of the distribution overlap is not a sufficient quantification of the ability of a given metric to differentiate between supercell and nonsupercell environments. Thus, while capable of more concretely illuminating the dependence of UTSRF on BWD, the previous analysis does not satisfactorily address the true merit in using UTSRF alone (i.e., controlling for BWD) to differentiate between supercell and nonsupercell environments.

To more accurately quantify the merit in using UTSRF alone, the probability of a supercell event [cf. (2)] will be examined. Because the number of supercell and nonsupercell events will be constrained by the range of BWD values used to control for BWD, (2) must be modified as follows:

$$
100 \cdot \frac{\mathrm{ns}\left(m_{B}, R_{B}, m_{U}, R_{U}\right)}{\mathrm{ns}\left(m_{B}, R_{B}, m_{U}, R_{U}\right)+\operatorname{nns}\left(m_{B}, R_{B}, m_{U}, R_{U}, A\right)},
$$

where $m_{U}$ and $R_{U}$ are the midpoint value and range for the UTSRF. For this analysis, $m_{B}$ and $R_{B}$ will be fixed and will depend on the depth of BWD that is being controlled for (e.g., as in Fig. 8, the midpoint value and range for $0-5-\mathrm{km}$ BWD are 13.5 and $\pm 2.5 \mathrm{~m} \mathrm{~s}^{-1}$, respectively). The value of $R_{U}$ will be fixed at $\pm 2.5 \mathrm{~m} \mathrm{~s}^{-1}$ and results will be plotted as a function of $m_{U}$.

To establish a benchmark for UTSRF, values of (3) are plotted in Fig. 9 as a function of $m_{U}$ without controlling for BWD (i.e., $R_{B}= \pm \infty$ ). Figure 9 illustrates that for each ratio, the likelihood of a supercell event is generally proportional to UTSRF. However, it is also clear from Fig. 9 that for ratios less than or equal to 1-2 (i.e., 1-5, 1-20, etc) the likelihood of a supercell event never exceeds the likelihood of a nonsupercell event. Thus, for no value of UTSRF at these ratios can a 


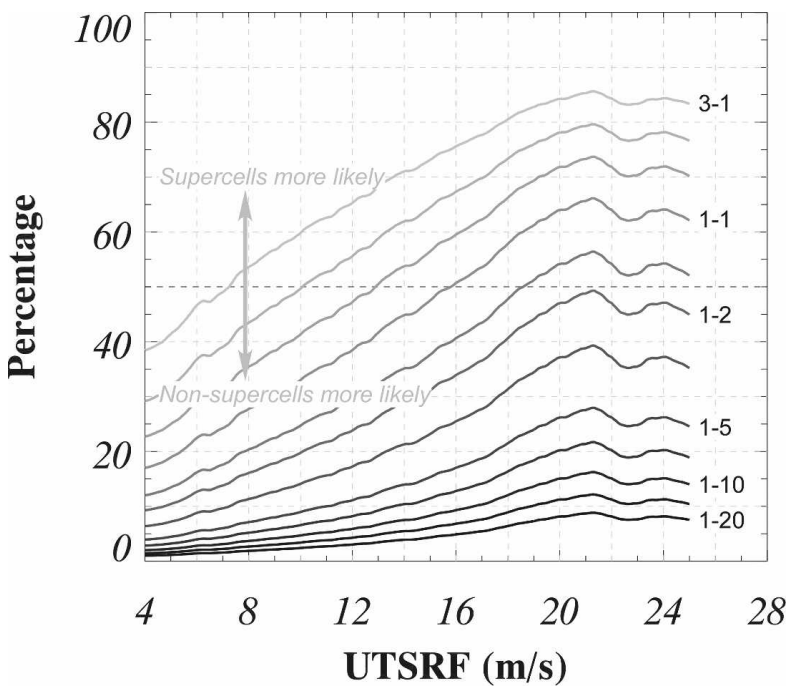

FIG. 9. As in Fig. 6 but for UTSRF. supercell event be expected to occur more frequently than a nonsupercell event. Consequently, if the actual ratio of supercell events to nonsupercell events equals or exceeds $1-2$, there is little merit in using the UTSRF to forecast supercells. This result can be anticipated from the overlap in the supercell and nonsupercell distributions illustrated in Fig. 7 but it is only through the preceding analysis that such a finding can be quantified.

This finding can be extended to the analysis of UTSRF when controlling for BWD. The increase in distribution overlap identified previously and illustrated in Fig. 8 suggests that removing the dependence of UTSRF on BWD will reduce the ability of UTSRF to delineate supercell from nonsupercell environments. An evaluation of (3) for UTSRF while controlling for BWDs through layers of $0-4,0-5,0-6$, and $0-7 \mathrm{~km}$ is illustrated is Fig. 10. As was the case when not controlling for BWD (cf. Fig. 9), for small ratios a nonsupercell event will be expected to occur more frequently than a

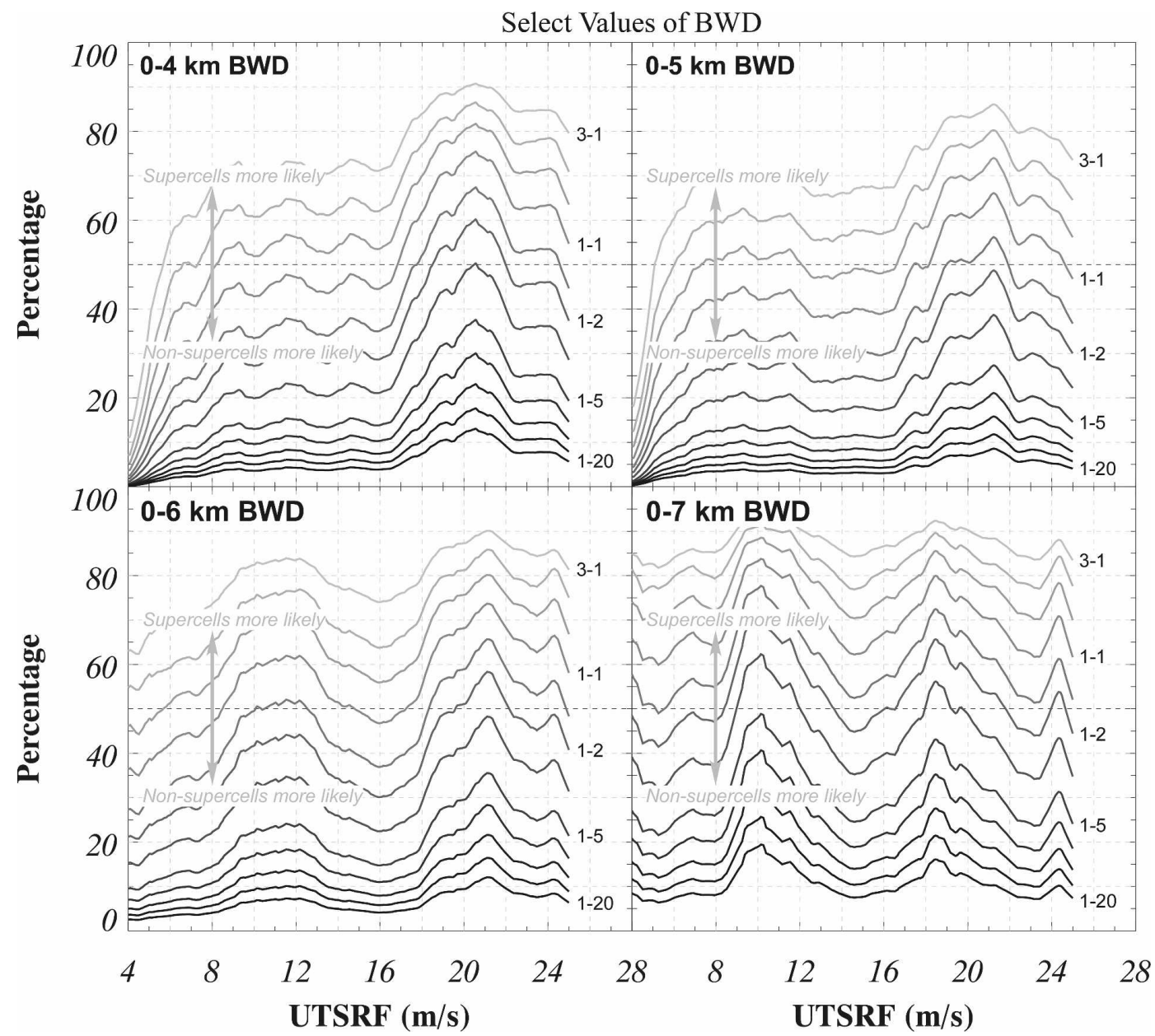

FIG. 10. As in Fig. 6 but for UTSRF at the select ranges of BWD used in Fig. 8. 
supercell event for all values of UTSRF. Moreover, when controlling for BWDs through layers of 0-6 and 0-7 km, the larger ratios yield probability distributions in which a nonsupercell event will never be expected to occur more frequently than a supercell event for all values of UTSRF. For those probability distributions that possess a threshold value (i.e., distributions that cross the $50 \%$ line), more than half do not exhibit a consistent relationship between the value of UTSRF and the likelihood of a supercell relative to a nonsupercell. For example, the probability distribution for a ratio of 1-1 when controlling for the $0-6-\mathrm{km}$ BWD indicates that nonsupercells are more likely than supercells for UTSRF values between 4 and $8 \mathrm{~m} \mathrm{~s}^{-1}$ and between 15 and $17 \mathrm{~m} \mathrm{~s}^{-1}$ and that supercells are more likely than nonsupercells for UTSRF values between 8 and 16 $\mathrm{m} \mathrm{s}^{-1}$ and for values greater than $17 \mathrm{~m} \mathrm{~s}^{-1}$. This inconsistent trend and the presence of multiple threshold values coupled with the observation that, for small ratios, a nonsupercell event will be expected to occur more frequently than a supercell event for all values of UTSRF indicates that the UTSRF, particularly when isolated from the BWD, is an unreliable metric for forecasting supercell events.

\section{Conclusions}

The aim of this article was to identify the depth of the layer over which the (fixed layer) BWD best discriminates between supercell and nonsupercell environments, and to test the ability of the UTSRF to make the same discrimination. Analysis relied on a dataset of soundings derived from RUC-2 analysis data interpolated to the inflow of radar-identified supercell and nonsupercell storms observed during two data collection periods totaling $4 \mathrm{yr}$.

Previous climatologies of sounding-based supercell forecast parameters have documented the ability of the 0-6-km BWD in delineating supercell from nonsupercell environments. However, a systematic examination of a wide range of layers has never been documented. This analysis revealed that the BWD computed for any of the layers between 0-4 and 0-6 km discriminates well between supercell and nonsupercell environments but that it is the BWD within the $0-5-\mathrm{km}$ layer that delineates best between supercell and nonsupercell environments.

In an attempt to identify possible threshold values of the $0-5-\mathrm{km}$ BWD that can be used to delineate supercell from nonsupercell environments, the probability of finding a supercell event among the complete dataset was examined. Accurately conducting this analysis required approximating the actual ratio of supercell to

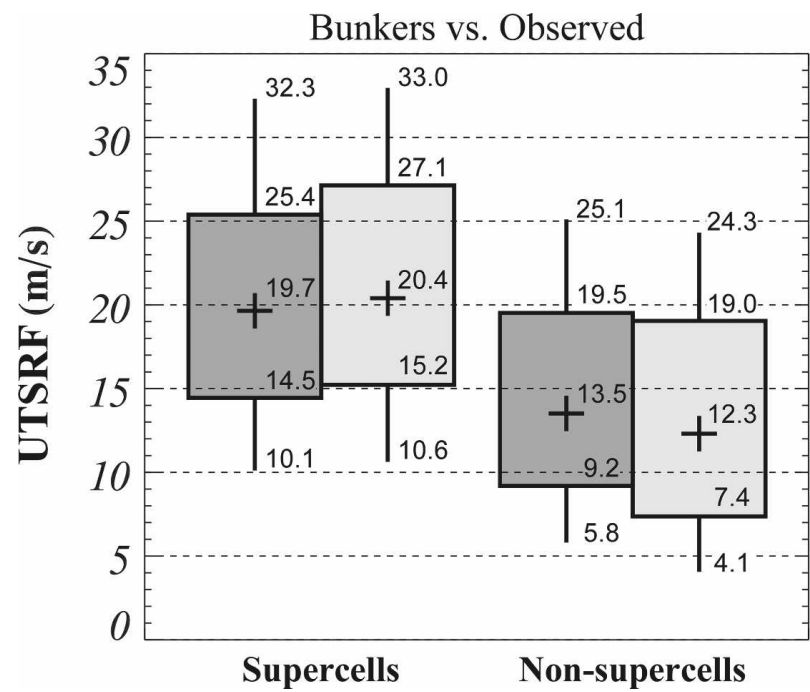

FIG. A1. As in Fig. 7 but with the UTSRF computed using the observed storm motion (light gray boxes) included.

nonsupercell events. This analysis demonstrated that for a 1-1 ratio (equal number of supercell and nonsupercell events) the threshold value of the $0-5-\mathrm{km}$ BWD would be $\sim 14 \mathrm{~m} \mathrm{~s}^{-1}$. However, if the actual ratio is closer to 1-20 (20 times more nonsupercell events than supercell events), then the threshold would be $\sim 20 \mathrm{~m} \mathrm{~s}^{-1}$.

The upper-tropospheric storm-relative flow has been offered previously as a sounding-based forecast parameter that could discriminate between supercell and nonsupercell environments. However, by examining the probability of finding a supercell event among the entire dataset, it was demonstrated that if the actual supercell to nonsupercell ratio is less than or equal to $1-2$, the UTSRF would have no ability to discriminate between environments that are more likely to support supercell events from environments that are more likely to support nonsupercell events. The lack of merit in using the UTSRF to forecast supercells is particularly evident when the UTSRF is isolated from the BWD. Because the UTSRF and BWD are not independent, controlling for the BWD when examining the UTSRF reveals that the UTSRF is not a fundamental parameter that can be used to distinguish supercell from nonsupercell environments. Therefore, this work demonstrated that the UTSRF is an unreliable metric for forecasting supercell events.

Acknowledgments. The authors are grateful for the thorough reviews of three anonymous reviewers whose constructive criticisms helped improve the manuscript. 


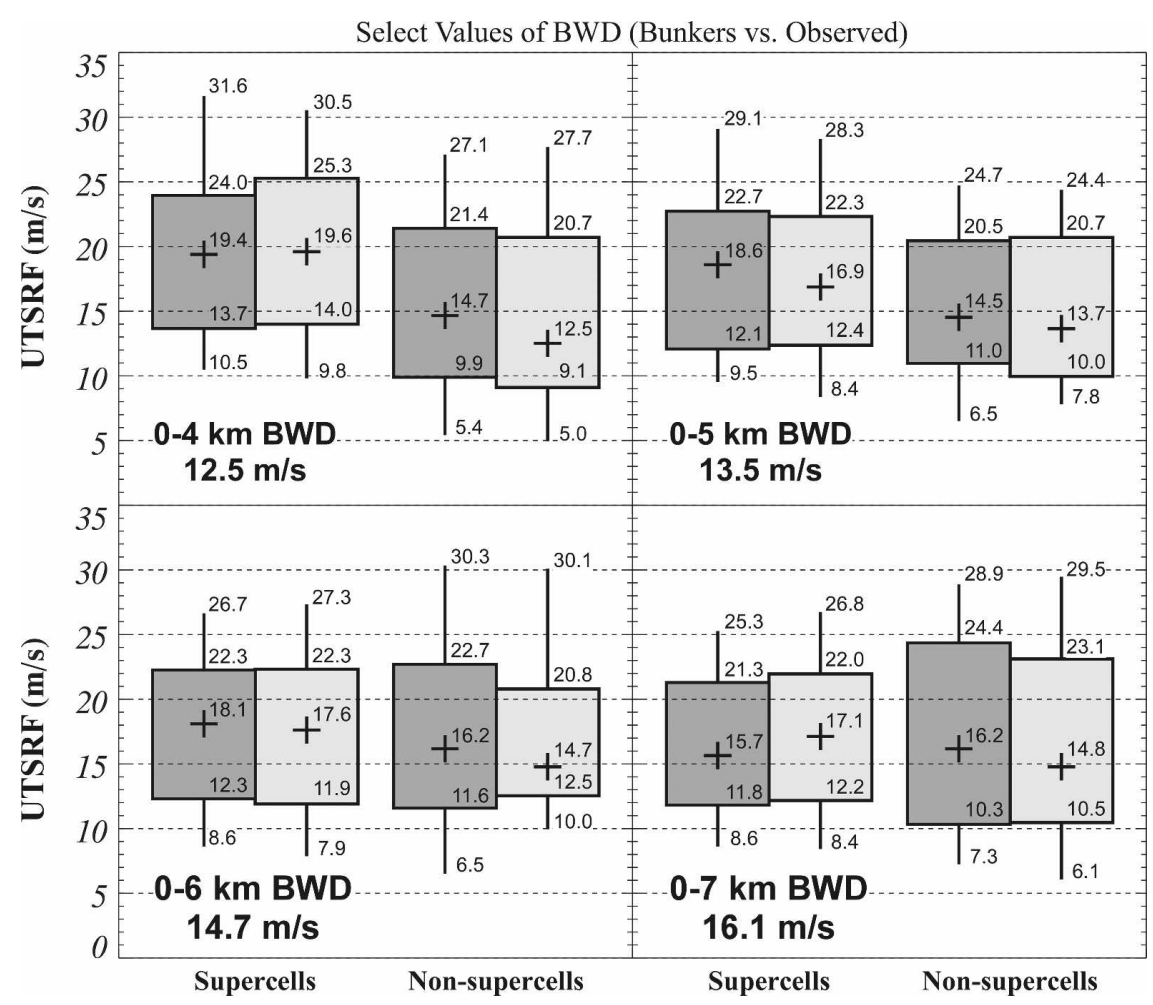

FIG. A2. As in Fig. A1 except that only specific ranges of BWD are considered (the BWD layer and midpoint value of the BWD range is listed at the bottom left of each panel).

\section{APPENDIX}

\section{Use of Observed Storm Motion for UTSRF}

In section 4 , the ability of the upper-tropospheric storm-relative flow (UTSRF) to discriminate between the environments of supercells and nonsupercells was examined using UTSRFs computed based on storm motions predicted with the Bunkers method. The boxand-whisker analysis of UTSRFs is repeated here for the UTSRFs computed using the observed storm motion instead. Comparison of the results using each type of storm motion is illustrated in Fig. A1 and A2. The similarities between the two results indicate that the same observations made based on the UTSRFs using the predicted storm motion can be made when using observed storm motion; namely, 1) supercells tend to occupy environments with larger values of UTSRF, 2) the overlap in the distributions is very large, and 3) when controlling for the BWD, the separation between the supercell and nonsupercell distributions decreases as the BWD-layer depth increases from 4 to $7 \mathrm{~km}$. Thus, using the observed storm motion instead of the predicted storm motion to compute the UTSRF does not alter the conclusion that the discriminating ability of UTSRF is largely tied to the discriminating ability of the BWD.

\section{REFERENCES}

Benjamin, S. G., and Coauthors, 2004: An hourly assimilation cycle: The RUC. Mon. Wea. Rev., 132, 495-518.

Bunkers, M. J., B. A. Klimowski, J. W. Zeitler, R. L. Thompson, and M. L. Weisman, 2000: Predicting supercell motion using a new hodograph technique. Wea. Forecasting, 15, 61-79.

Craven, J. P., H. E. Brooks, and J. A. Hart, 2002: Baseline climatology of sounding derived parameters associated with deep, moist convection. Preprints, 21st Conf. on Severe Local Storms, San Antonio, TX, Amer. Meteor. Soc., 643-646.

Droegemeier, K. K., S. M. Lazarus, and R. P. Davies-Jones, 1993: The influence of helicity on numerically simulated convective storms. Mon. Wea. Rev., 121, 2005-2029.

Fawbush, E. J., and R. C. Miller, 1952: A mean sounding representative of the tornadic airmass environment. Bull. Amer. Meteor. Soc., 33, 303-307.

— , and - 1954: The types of airmasses in which North American tornadoes form. Bull. Amer. Meteor. Soc., 35, 154165.

,-- , and L. G. Starrett, 1957: Severe local storms and midtropospheric flow patterns. Bull. Amer. Meteor. Soc., 38, 115123.

Markowski, P. M., C. Hannon, J. Frame, E. Lancaster, A. Pietrycha, R. Edwards, and R. L. Thompson, 2003: Characteristics 
of vertical wind profiles near supercells obtained from the Rapid Update Cycle. Wea. Forecasting, 18, 1262-1272.

Marwitz, J. D., 1972: The structure and motion of severe hailstorms. Part I: Supercell storms. J. Appl. Meteor., 11, 166-179.

McCaul, E. W., Jr., 1991: Buoyancy and shear characteristics of hurricane tornado environments. Mon. Wea. Rev., 119, 19541978.

Moncrieff, M. W., and J. S. A. Green, 1972: The propagation and transfer properties of steady convective overturning in shear. Quart. J. Roy. Meteor. Soc., 98, 336-352.

Ramsay, H. A., and C. A. Doswell III, 2005: A sensitivity study of hodograph-based methods for estimating supercell motion. Wea. Forecasting, 20, 954-970.

Rasmussen, E. N., and R. B. Wilhelmson, 1983: Relationship between storm characteristics and 1200 GMT hodographs, lowlevel shear, and stability. Preprints, 13th Conf. on Severe Local Storms, Tulsa, OK, Amer. Meteor. Soc., J5-J8.

_- and D. O. Blanchard, 1998: A baseline climatology of sounding-derived supercell and tornado forecast parameters. Wea. Forecasting, 13, 1148-1164.

Thompson, R. L., R. Edwards, J. A. Hart, K. L. Elmore, and P. M. Markowski, 2003: Close proximity soundings within supercell environments obtained from the Rapid Update Cycle. Wea. Forecasting, 18, 1243-1261.

_ C. M. Mead, and R. Edwards, 2007: Effective storm-relative helicity and bulk shear in supercell thunderstorm environments. Wea. Forecasting, 22, 102-115.

Weisman, M. L., and J. B. Klemp, 1982: The dependence of numerically simulated convective storms on vertical wind shear and buoyancy. Mon. Wea. Rev., 110, 504-520.

- , and - 1984: The structure and classification of numerically simulated convective storms in directionally varying wind shears. Mon. Wea. Rev., 112, 2479-2498.

—_, and — 1986: Characteristics of isolated convective storms. Mesoscale Meteorology and Forecasting, P. Ray, Ed., Amer. Meteor. Soc., 331-358. 